Document downloaded from:

http://hdl.handle.net/10251/120930

This paper must be cited as:

Lerma, JL.; Barbero-García, I.; Marqués-Mateu, A.; Miranda, P. (2018). Smartphone-based video for 3D modelling: Application to infants cranial deformation analysis. Measurement. 116:299-306. https://doi.org/10.1016/j.measurement.2017.11.019

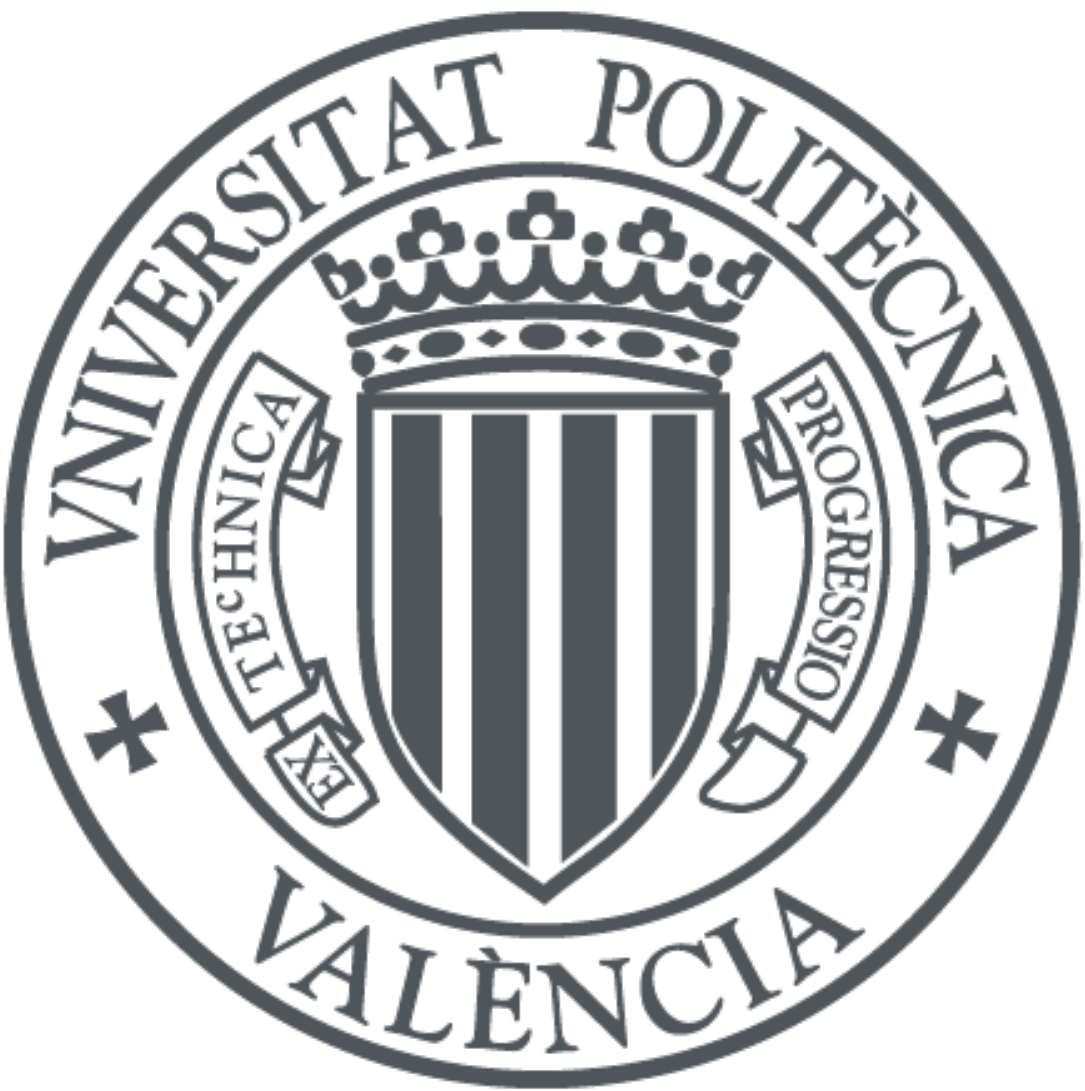

The final publication is available at

https://doi.org/10.1016/j.measurement.2017.11.019

Copyright Elsevier

Additional Information 


\section{SMARTPHONE-BASED VIDEO FOR 3D MODELLING: APPLICATION TO INFANT'S CRANIAL DEFORMATION ANALYSIS}

José Luis Lerma ${ }^{1}$, Inés Barbero-García ${ }^{1,}$ Ángel Marqués-Mateu ${ }^{1}$, Pablo Miranda ${ }^{2}$

${ }^{1}$ Photogrammetry \& Laser Scanning Research Group (GIFLE), Department of Cartographic Engineering, Geodesy and Photogrammetry, Universitat Politècnica de València, Camino de Vera, s/n, Building 7i. 46022 Valencia, Spain

${ }^{2}$ Hospital Universitari i Politècnic/Instituto de Investigación Sanitaria La Fe

\section{Corresponding author:}

Inés Barbero-García. inbargar@topo.upv.es

Photogrammetry \& Laser Scanning Research Group (GIFLE), Department of Cartographic Engineering, Geodesy and Photogrammetry, Universitat Politècnica de València, Camino de Vera, s/n, Building 7i. 46022 Valencia, Spain

\section{Abstract}

The use of smartphones cameras for photogrammetric purposes is increasing. However, the suitability of smartphones for 3D modelling for medical purposes in general, and for cranial deformation in particular, is still to be analysed. This paper investigates the suitability of smartphone video cameras to create $3 D$ models for cranial deformation analysis compared to the digital single-lens reflex (SLR) cameras traditionally used in close-range photogrammetry. Two models are obtained, the first one from a slow-motion video recorded with a smartphone, and the second one from SLR camera imagery. The models are compared to evaluate the differences not only between themselves but also through the best fitting ellipsoid that allow the determination of the cranial deformations. The average distance between models is $0.5 \mathrm{~mm}$, and below $1 \mathrm{~mm}$ for $86 \%$ of the model points. The maximum difference between the two fitted ellipsoid semiaxes is $1 \mathrm{~mm}$. It can be stated that smartphones are a low-cost solution that can provide $3 D$ 
models with a similar accuracy to that of SLR cameras for non-static objects in close range scenarios. More interestingly, slow-motion videos provide comparable results in real clinical conditions with infants in movement.

Keywords: close-range photogrammetry, medicine, evaluation, videogrammetry.

\section{INTRODUCTION}

Cranial deformation in infants is a group of conditions that affects a great percentage of new-borns (Rogers, 2011). Although its prevalence is high, there are no standards for its systematic evaluation. Usually, the deformation is measured using callipers but experts do not fully agree on the suitability of this methodology. Photogrammetry is another of the main techniques in use (Siegenthaler, 2015), but it is not widely extended in consultations and hospitals despite its suitability to carry out non-contact and accurate measurements.

Image-based 3D digitisation and 3D modelling are being used for a wide range of medical applications such as the creation of prosthesis (SalazarGamarra et al., 2016), diagnosis (Farnood Ahmadi and Layegh, 2014), craniofacial information analysis (Byvaltsev, Belykh and Belykh, 2012) and dental reconstruction (Grenness et al. 2005; Hernandez and Lemaire 2016). However, the most common tools to obtain 3D information for medical purposes are magnetic resonance imaging (MRI) and computed tomography (CT) scanners. These methods are still considered the most reliable options and are the only ones to provide inner tissue information. In spite of their possibilities, MRI and TC scanners are costly and highly invasive. Its use in infants usually requires the use of anaesthesia to put the patient to sleep. Another approach for the extraction of 3D data is the use of special 3D scanners such as the STARscanner (Plank et al., 2006). These tools are applied for the creation of the orthotic helmets used to correct the deformations as well as an analysis tool. The main issue of this methodology is the high cost of the devices.

Photogrammetry and 3D modelling are non-invasive and provide more detailed data than calliper measurements. However, the cost of the photogrammetric processes depends highly on the setup. One of the main challenges for the measurement of cranial deformation in infants is the movement of the patients. Infants, between 2 and 8 months old are, in most cases, in constant movement during the consultation, and below three months, they can hardly hold their heads. Therefore, special care must be taken to assure that the images are well-focused. The use of SLR professional cameras, although possible in some cases (i.e. the infant is extremely calmed), is usually challenging, it requires time and additional 
lighting sources. On the other hand, the use of a single portable triangulation 3D scanner, such as Sense 3D (3D Systems, U.S.), is even more limited by the infant's movement and does not work with this type of real-life settings.

To deal with the problem of the movement, some photogrammetric techniques include complex setups of several professional cameras or a combination of cameras and 3D scanners. For these solutions, costly equipment is required. The use of smartphone cameras as a tool to create high-accuracy 3D models is becoming more common as the capabilities of the smartphones increase and they are equipped with higher quality cameras (Daponte et al., 2013; Bakula and Flasiński, 2014). Smartphone-based photogrammetry is starting to be used in different medical studies, most of them focused on the creation and measurement of medical prosthesis (Salazar-Gamarra et al. 2016; Hernandez and Lemaire 2016). However, in these studies, the target is static and photographs are used instead of video sequences. The use of videogrammetry, an imaging technique that extracts 3D measures from video, is also becoming more common as it allows the acquisition of a large number of well-focused images in short periods of time, even with moving targets (Chong, 2012). Videogrammetry is usually understood as the science to extract 3D coordinates as a function of time, allowing the obtainment of multitemporal data of dynamic objects (Black and Pappa 2003; Lin et al. 2008). In this particular study, there is no intention to obtain dynamic information but well-focused images of a non-changing moving target. Videogrammetry reduces the time required for image acquisition and the high number of frames allows the removal of bad focused images (Xu et al., 2016). Smartphone-based slow-motion videos are an especially interesting methodology which allow us to obtain focused images of moving targets easily.

The aim of this article is to compare the results obtained using photographs taken with a digital SLR camera and those obtained using a slow-motion video recorded with a smartphone by means of an auxiliary ellipsoid shape fitting the head. The use of a Sense 3D scanner was also part of the initial experiment. However, it was not possible to obtain a model of the infant using this device and therefore, the results achieved with the triangulation laser scanner are not reported here. Nevertheless, the experiments undertaken will indicate whether slow motion videos taken with a smartphone can provide a similar accuracy than SLR cameras for the measurement of cranial deformation in infants or not.

\section{METHODOLOGY}

Two 3D models of the head were created for the same patient using two imaging sensors: an Advanced Photo System type-C (APS-C) frame SLR 
camera and a high-end smartphone in slow-motion video mode; a set of frames was later extracted from the video. Parallel processes were carried out to create both models. The images were masked and orientation and selfcalibration processes were carried out. Once each mesh was obtained, it was cleaned and smoothed. Lastly, the fitted ellipsoid was calculated for each mesh using least squares adjustment and the ellipsoids and meshes were compared to evaluate their differences (Fig. 1).

The acquisition of well-focused images to create the 3D model using the SLR camera was possible due to a situation more favourable than usual (i.e. the infant was specially calmed). Nevertheless, the image acquisition lasted longer than the video recording. 


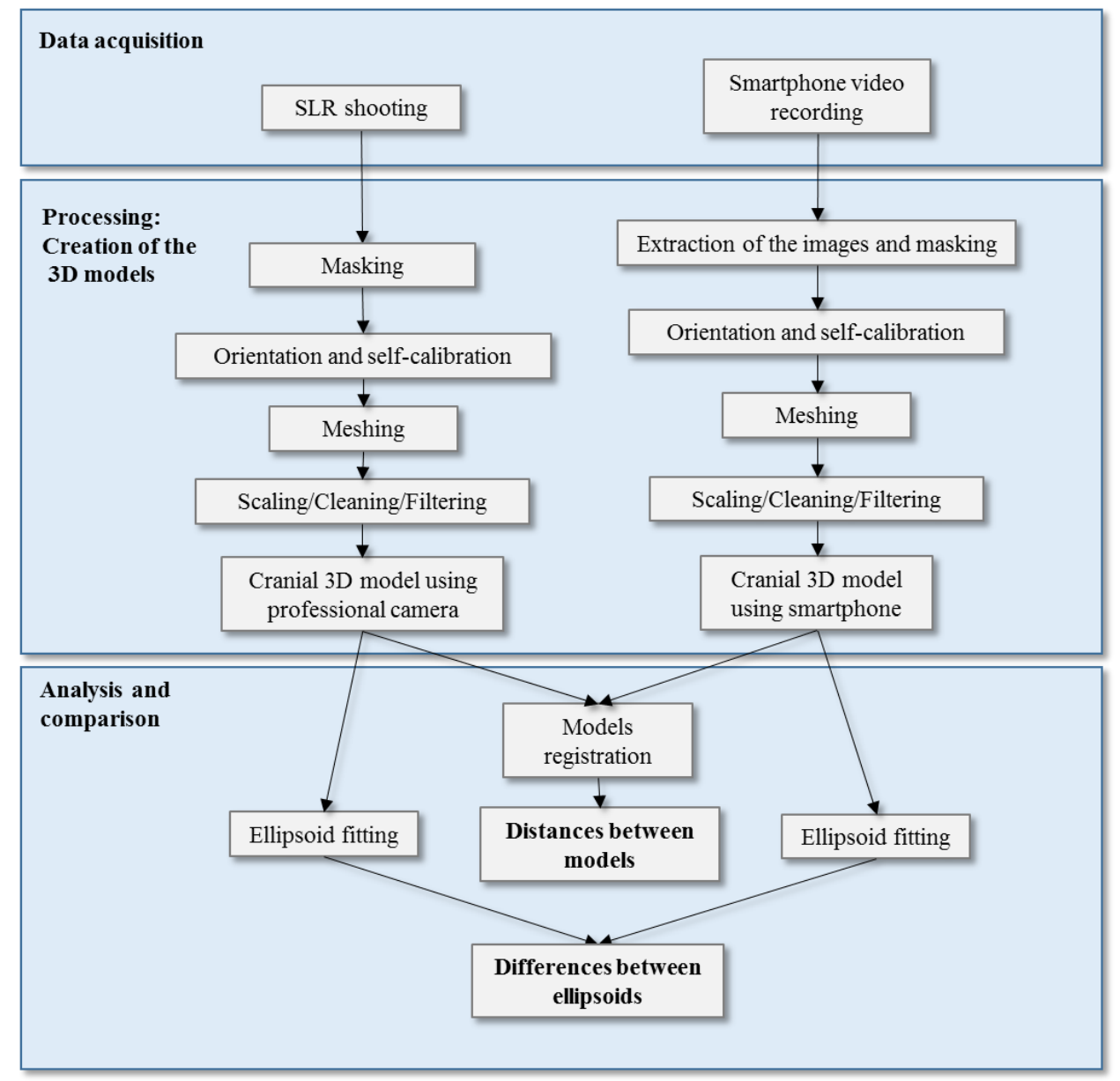

Fig. 1. Flow diagram.

\section{Setup}

The image acquisition was carried out during a standard medical consultation, which involves several limitations. The time slot is very limited as the doctor has to move to the next patient within minutes. The infant (normally aged between 2 and 8 months old) is usually awake and moves quickly during the process and, in many cases, he gets nervous when he is set on an ideal position to take the pictures. In addition, patient's parents are usually present and a certain degree of nervousness is to be expected. In 
this situation, it is vital to be quick, straightforward and interfere the minimum with the consultation.

The setup for the data acquisition was therefore kept as simple as possible. This assures that the cost related to imaging equipment is expected to be low and the processes can be carried out regardless of the consultation place. The image shooting and the video recording were carried out during the same consultation. The infant was hold by an adult, therefore no special equipment was required. A cap was placed on the children's head to avoid hair from affecting the quality of the model. Four pieces of measuring tape, of approximately two centimetres were placed on the cap. These marks were included in the model to allow scaling the model and to facilitate the image registration with easily identifiable features.

In order to simplify the process no special light conditions were required, the usual illumination of the consultation room was enough.

\section{Data acquisition}

Two imaging devices were used for the data acquisition (Fig. 2). First, a digital SLR camera Canon EOS 1100D was used at a maximum resolution of $4272 \times 2848$ pixels with a Canon lens EF-S $18-55 \mathrm{~mm}$. A principal distance of $35 \mathrm{~mm}$ was selected for taking the pictures at ISO 3200. No tripod or special illumination was used. Second, a high-end smartphone Samsung Galaxy S7 Edge (Fig. 2b) in slow-motion video was used at a resolution of $1280 \times 738$ pixels (Table 1 ).

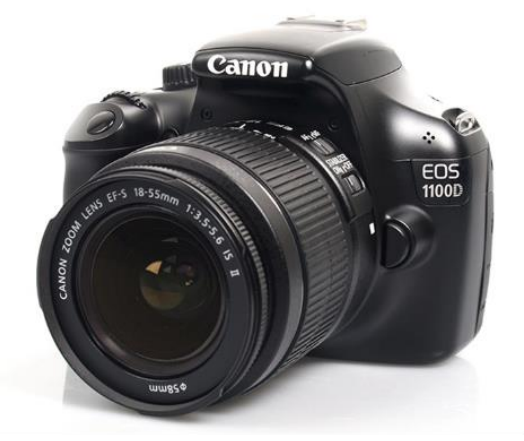

(a)

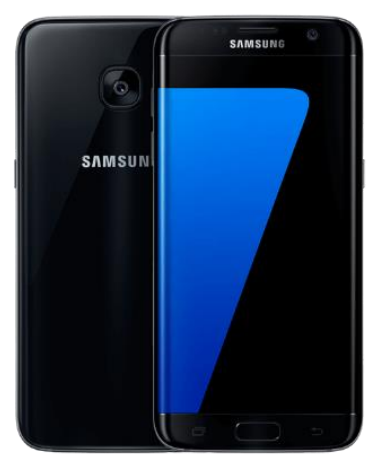

(b)

Fig. 2. Cameras used during the image acquisition: (a) Canon EOS 1100D, and (b) Samsung Galaxy S7 Edge. 


\begin{tabular}{lcccc}
\hline Camera & $\begin{array}{c}\text { Focal } \\
\text { length } \\
(\mathrm{mm})\end{array}$ & $\begin{array}{c}\text { Effective } \\
\text { format size } \\
(\mathrm{mm})\end{array}$ & $\begin{array}{c}\text { Pixel count } \\
\text { (pixels) }\end{array}$ & $\begin{array}{c}\text { Pixel pitch } \\
(\mathrm{mm})\end{array}$ \\
\hline $\begin{array}{l}\text { Canon EOS } \\
1100 \mathrm{D}\end{array}$ & 34.35 & $22.17 \times 14.78$ & $4272 \times 2848$ & 0.00519 \\
$\begin{array}{l}\text { Samsung } \\
\text { Galaxy S7 }\end{array}$ & 4.2 & $5.64 \times 4.23$ & $\begin{array}{c}\text { Video: } 1280 \times 738 \\
\text { Still: } 4032 \times 3024\end{array}$ & 0.0014 \\
\hline
\end{tabular}

Table 1. Sensor specifications

It has been reported that good-quality 3D models can be obtained using approximately 10-20 images for outer dome surfaces (geometry close to the infant's head) using multi-convergent images (Kraus, 1997). In this study, a higher number of images was acquired to assure that the final model can be created. A high number of images was found to be necessary as the geometry of the network is not ideal for calibration (i.e. the infant's head occupies only the central part of the image). Another unexpected issue was that many of the images were blurred due to the quick infant's head movement. Fig. 3 displays a partial view of the two data sets.
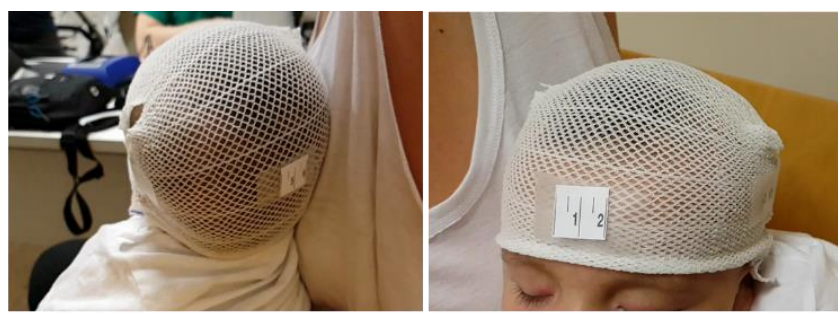

(a)
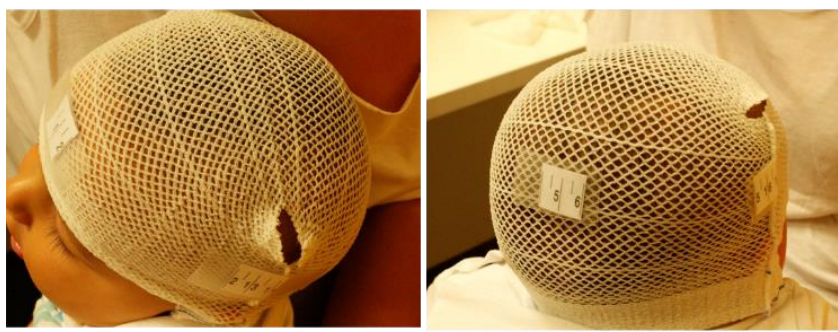

(b)
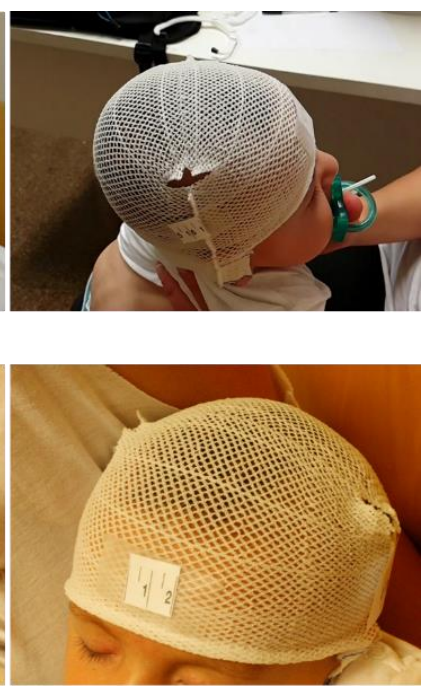

Fig. 3. Sample of images used for the processing and 3D modelling: (a) extracted from the slow-motion video; (b) taken with SLR camera. 


\section{Processing}

The two data sets were processed independently using Photoscan Professional version 1.2.6 (Agisoft LLC, Russia).

The first dataset was composed by the images taken with the SLR camera once the blurry images were discarded. A large number of images were obtained as the elimination rate was expected to be high due to the head movement. Finally, 24 images out of 53 were used.

The second set was composed of the images extracted from the video. The extraction of the most useful frames from the video is one of the main challenges in videogrammetry. The usage of the totality of the images is unnecessary and would be unmanageable in terms of computational cost. The selected images must be well-focused and present a good geometry around the object. In this case, a set of images were extracted automatically using a fixed frequency (e.g. one frame out of ten). Later, the blurred images were automatically removed using an automated procedure. The individual image quality was estimated and the bad-quality images were determined using a threshold. Finally, the geometry of the network was reviewed to manually remove redundant images.

In the end, a total of 63 frames were used for the 3D modelling from the second set. The quantity of frames is almost three times larger than the number of images used for the SLR camera model. A larger number of images is necessary a priori to compensate both the lack of resolution and the geometric accuracy of the camera.

The two sets of images followed the same steps to create the 3D models (Processing part, Fig. 1). Firstly, the images were masked to delimit the part of the images that were to be used for modelling. This process is necessary as the infant is moving in relation to the background. For that reason, it would not be possible to obtain an acceptable model without excluding this background through the image masks. The masking process was carried out manually, although the automation of the process must be approached in the future.

The second step was the image orientation (also known as alignment), which is the process used to determine the positions and rotations for each image in the object space. During this step, the cameras were also calibrated (see below the Results Section). A relative ground reference system (GRS) for the models was set by assigning coordinates to three points of known distances. This process was carried out automatically using Agisoft PhotoScan. Later, the point cloud was densified and the mesh created. The meshes needed to be cleaned and smoothed in order to remove the texture of the cap and possible artefacts. Lastly, the final models were scaled using the markers placed on the cap and the texture was draped on the 3D models (Fig. 4) 


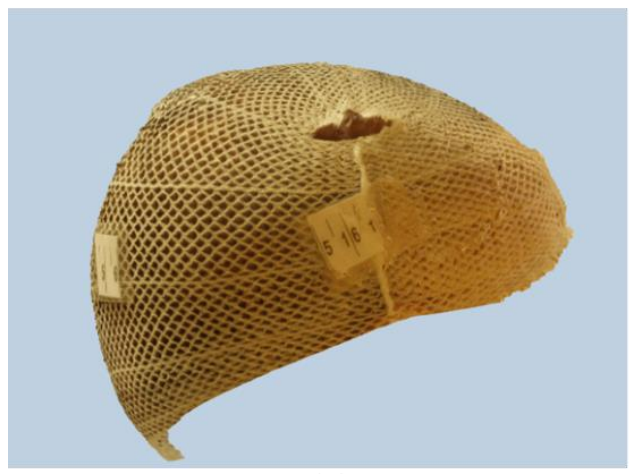

(a)

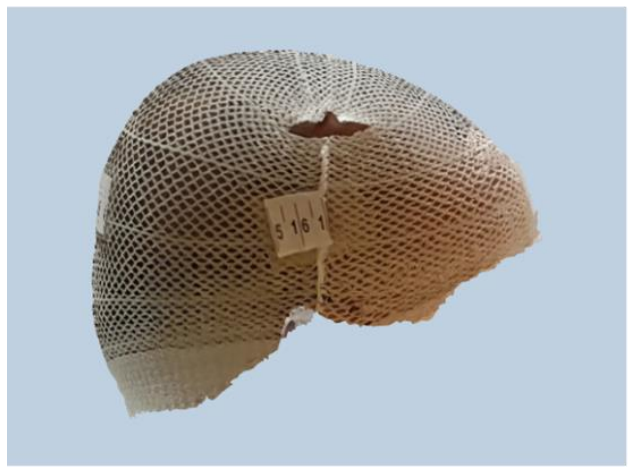

(c)

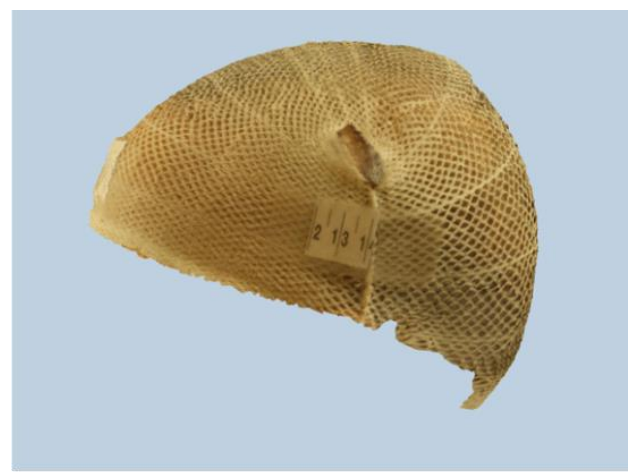

(b)

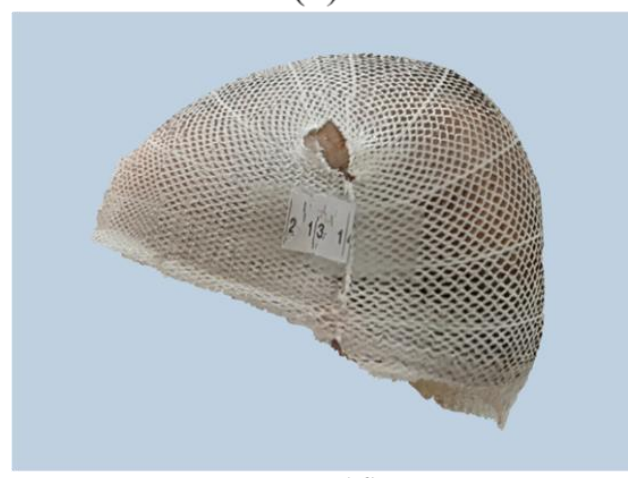

(d)

Fig. 4. 3D models created using both cameras: (a) right and (b) left views, Canon EOS 1100D ; (c) right and (d) left views, smartphone video.

\section{Self-calibration}

No independent calibration of the cameras was carried out as it would make the setup more complex and would greatly increase the time required by the doctor to collect the necessary data. Instead, a self-calibration process was undertaken with ten additional parameters. The self-calibration accuracy is expected to be limited by the nature of the setup. In most images, the object covers only a small area in the centre of the image, while the rest of the image must be masked. The inner instability of the smartphone camera is also expected to affect the calibration.

\section{Ellipsoid fitting}

The similarity in shape and volume between the human skull and the ellipsoid has been pointed out in previous studies (Bergerhoff, 1957). According to this 
study, the cranial deformation can be measured in terms of distances from the real head surface to an ellipsoid representing the ideal head surface (Barbero-García et al., 2017).

The ideal head shape will be different for each patient and, therefore, the ellipsoid must be calculated for each one. The ideal ellipsoid is the one which minimises the distances to the real head while meeting some constraints to assure that the head is normal, i.e. antrophometric cranial indexes considered normal according to medical literature (Pindrik et al., 2016).

The ideal ellipsoid was obtained by least square fitting using the mathematical model presented in Bektas (2015). The obtained parameters are the semiaxes of the fitted ellipsoid. The accuracy of the solution can be evaluated using the semiaxes standard deviation (Table 4). Approximately 30000 points from each model were used for the fitting process.

\section{Comparison}

The estimated accuracy was calculated for both cameras (Table 2) using the formula presented in Fraser (1992):

$$
\sigma=\frac{q}{\sqrt{k}} S \sigma_{i m g}
$$

The theoretical precision $\sigma$ is affected by the image co-ordinate standard error $\sigma_{\text {img, }}$, the image scale $S$ and the number of images $k$. The factor $\sigma_{\text {img }}$ is obtained as the maximum reprojection error provided by Agisoft PhotoScan.

The design factor $q$ is given by the strength of the camera network. For acceptable convergent networks, it is expected to take values from 0.4 to 0.8 . Values of 0.6 and 0.7 are considered suitable for design purposes in these cases (Fraser, 1996). A value of 0.6 has been assigned for both models as the network geometry is convergent. Since the goal of the study is the comparison of cameras and not the retrieval of absolute accuracy values, the value of this parameter will not affect the results, as the network geometry is the same for both cameras.

Once both models were created they were compared to evaluate their differences. At this point, the models were roughly registered, however small differences could be expected as only 3 points were used to set the relative GRS. Then, the registration was improved after identifying homologous points between the models in CloudCompare 2.7.0. working environment (GPL software). Afterwards, the Iterative Closest Point algorithm was applied to achieve the best possible accuracy in the registration. After that, the same software was used to compute the Euclidean distances between both models (i.e. the model created using the smartphone and the model created using 
the SLR camera) (Fig. 6). Following this way, the differences between models can be evaluated for each point. The evaluation of these differences will allow us to state whether the models are comparable or not.

The differences between the fitted ellipsoids were compared as differences in their three semiaxes.

\section{RESULTS}

Table 2 shows the calibration parameters and their standard deviations for both cameras. The significance of the additional parameters was evaluated using the Student's t-test (Kraus, 1997; Lerma, 2002). The standard deviations achieved are extremely high for the smartphone camera. In fact, Student's t-test confirms that only the principal distance parameter (f) is reliable. High standard deviations discredit the mean parameters during the self-calibration, and thereby they should not be taken into account. For the SLR camera, standard deviations were considerable smaller. The radial correction parameter $k_{1}$ presented a low significance value and therefore it will not be taken into account.

\begin{tabular}{lcccccc}
\hline & \multicolumn{3}{c}{ Samsung Galaxy S7 } & \multicolumn{3}{c}{ Canon EOS 1100D } \\
\cline { 2 - 7 } & Mean & Std & t-Student & Mean & Std & t-Student \\
\hline $\mathrm{f}$ & 29.08 & 2.41 & $100 \%$ & 37.43 & 0.22 & $100 \%$ \\
$\mathrm{x}_{0}$ & 1.23 & 0.87 & $78.2 \%$ & 1.29 & 0.03 & $100 \%$ \\
$\mathrm{y}_{0}$ & 0.70 & 2.17 & $23.8 \%$ & -0.16 & 0.02 & $100 \%$ \\
$\mathrm{k}_{1}$ & $-4.2 \cdot 10^{-04}$ & $8.1 \cdot 10^{-04}$ & $36.8 \%$ & $-2.9 \cdot 10^{-05}$ & $2.2 \cdot 10^{-05}$ & $74.7 \%$ \\
$\mathrm{k}_{2}$ & $1.4 \cdot 10^{-06}$ & $4.9 \cdot 10^{-06}$ & $20.9 \%$ & $-2.9 \cdot 10^{-06}$ & $1.2 \cdot 10^{-07}$ & $100 \%$ \\
$\mathrm{k}_{3}$ & $-1.7 \cdot 10^{-09}$ & $7.5 \cdot 10^{-09}$ & $16.6 \%$ & $3.0 \cdot 10^{-09}$ & $1.7 \cdot 10^{-10}$ & $100 \%$ \\
$\mathrm{p}_{1}$ & $-4.2 \cdot 10^{-04}$ & $8.5 \cdot 10^{-04}$ & $34.9 \%$ & $2.6 \cdot 10^{-04}$ & $3.5 \cdot 10^{-06}$ & $100 \%$ \\
$\mathrm{p}_{2}$ & $-7.0 \cdot 10^{-04}$ & $9.5 \cdot 10^{-04}$ & $50.2 \%$ & $1.9 \cdot 10^{-04}$ & $6.2 \cdot 10^{-06}$ & $100 \%$ \\
$\mathrm{~b}_{1}$ & 0.04 & $9.4 \cdot 10^{-03}$ & $98.9 \%$ & $-2.8 \cdot 10^{-03}$ & $5.3 \cdot 10^{-05}$ & $100 \%$ \\
$\mathrm{~b}_{2}$ & $9.1 \cdot 10^{-03}$ & $8.5 \cdot 10^{-03}$ & $46.2 \%$ & $-4.9 \cdot 10^{-03}$ & $3.1 \cdot 10^{-04}$ & $100 \%$ \\
\hline
\end{tabular}

Table 2. Additional calibration parameters for the two cameras ( $\mathrm{f}, \mathrm{x} 0$, $\mathrm{y} 0 \mathrm{in} \mathrm{mm}$ ), standard deviations and significance percentage according to Student's t-test.

The great differences in the image deformations can be graphically observed in Fig. 5. As it may be expected, both radial and tangential distortions are much higher for the smartphone camera. In spite of these values, if the corrections are correctly calculated and applied, the final geometric camera calibration model will cope with the deformation and the observations will be free of systematic errors thanks to the interior orientation parameters. 


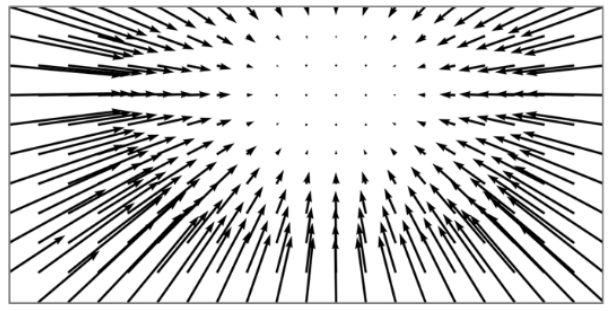

(a)

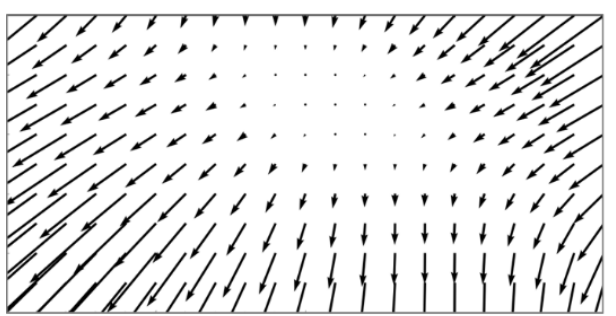

(c)

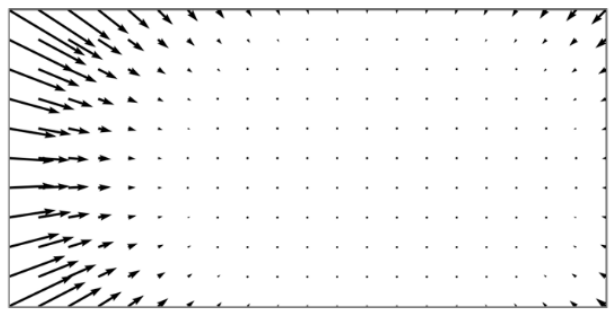

(b)

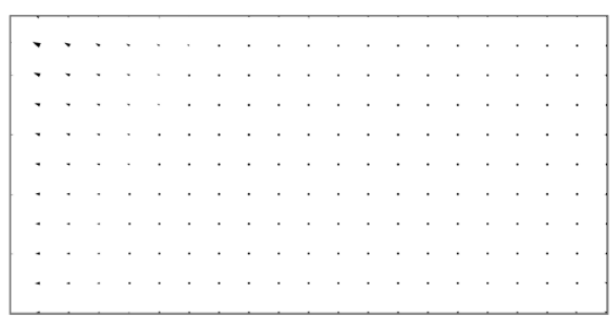

(d)

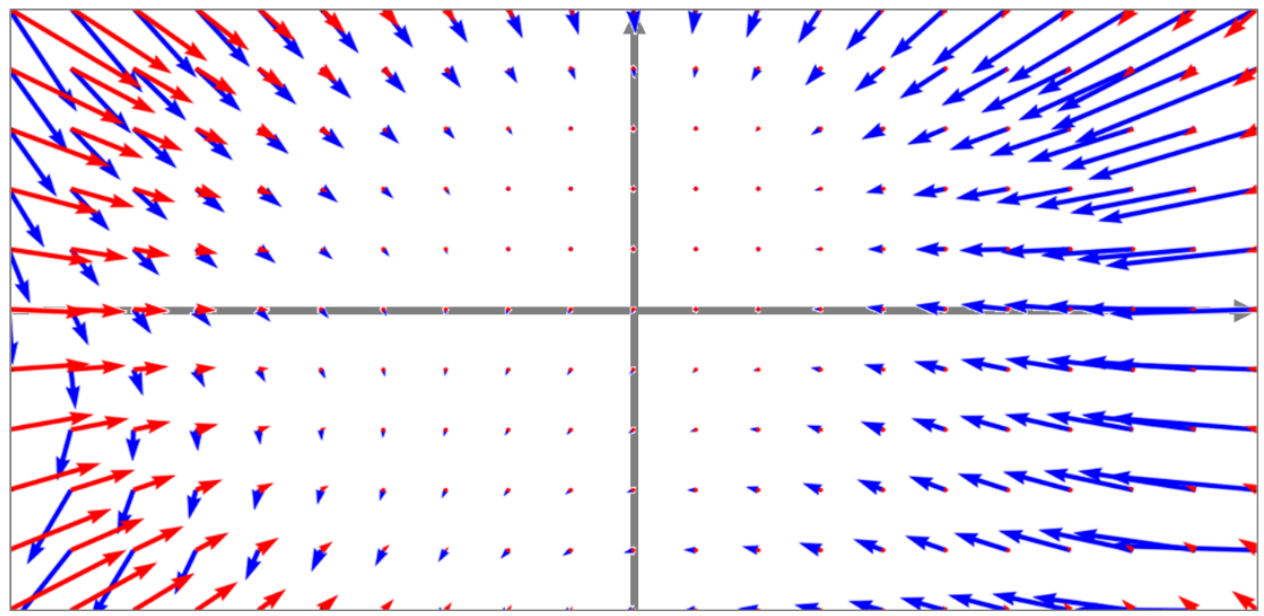

(e)

Fig. 5. Distortion grids: (a) Radial distortion for smartphone camera. (b) Radial distortion for SLR camera. (c) Tangential distortion for smartphone camera. (d) Tangential distortion for SLR camera. (e) Total distortion for both smartphone camera (blue) and SLR camera (red).

The theoretical precision and the relative accuracy, together with the parameters that affect them, can be checked in Table 3. The standard deviation of the image measurements $\sigma_{\text {img }}$ is slightly better for the SLR 
camera. Nevertheless, the final estimated precision is slightly better for the smartphone camera. This difference is caused mainly by the larger quantity of images obtained and used with the smartphone. The differences in precision can be considered not relevant.

\begin{tabular}{ccccccc}
\hline Camera & $\begin{array}{c}\text { Mean } \\
\text { distance } \\
(\mathrm{mm})\end{array}$ & $\begin{array}{c}\text { Design } \\
\text { factor } q\end{array}$ & $\begin{array}{c}\text { Number } \\
\text { of } \\
\text { images } \\
K\end{array}$ & $\begin{array}{c}\text { Standard } \\
\text { deviation of } \\
\text { image } \\
\text { measurements } \\
\text { oimg }(\mathrm{mm})\end{array}$ & $\begin{array}{c}\text { Theoretical } \\
\text { precision } \\
\sigma(\mathrm{mm})\end{array}$ & $\begin{array}{c}\text { Triangulation } \\
\text { accuracy } \\
\text { indicator } \sigma / R\end{array}$ \\
\hline $\begin{array}{c}\text { Canon } \\
\text { EOS } \\
\begin{array}{l}\text { 1100D } \\
\text { Galaxy }\end{array}\end{array}$ & 310 & 0.6 & 24 & 0.016 & 0.019 & $1: 80000$ \\
S7 Edge & 256 & 0.6 & 63 & 0.02 & 0.013 & $1: 110000$ \\
\hline
\end{tabular}

Table 3. Differences in estimated precision for both cameras.

Fig. 6 shows several views and a histogram with the Euclidean distances between the two 3D models. These distances are represented as hypsometric colours on the model created using the SLR camera. Some areas of notable difference can be distinguished; an important part of them being close to the edge of the cap or belonging to one of the markers that may have moved during the image acquisition. The figure also shows the histogram of the distances. The mean distance between models is $0.5 \mathrm{~mm}$, and the standard deviation $0.47 \mathrm{~mm}$. A small bias can be detected as the mean of the signed distances is $0.17 \mathrm{~mm}$ and the probability mass under zero is $41.6 \%$. Nevertheless, it is work noticing that $86 \%$ of the distances between both models are below $1 \mathrm{~mm}$. 


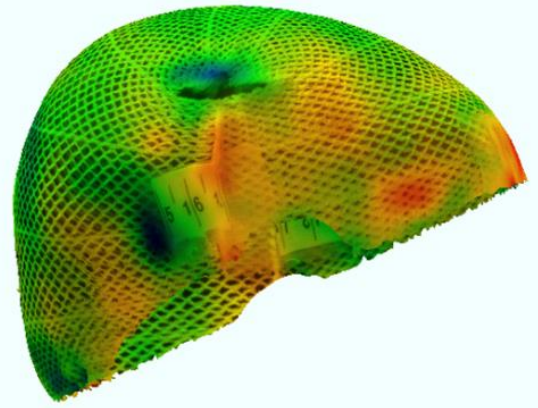

(a)

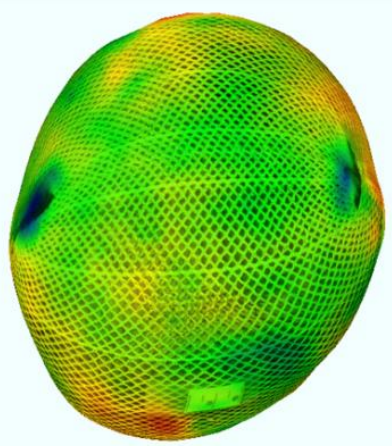

(c)

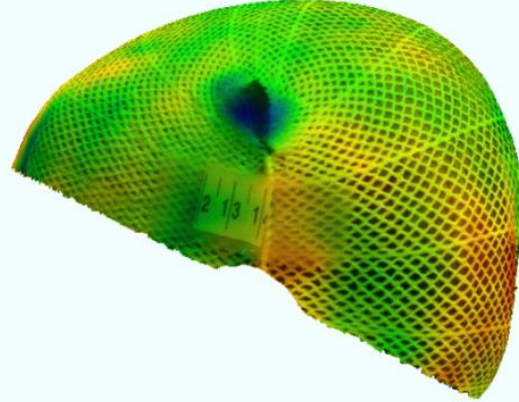

(b)

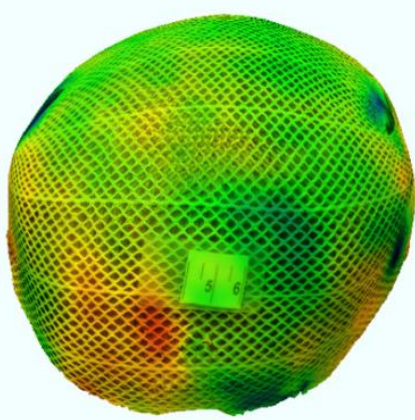

(d)

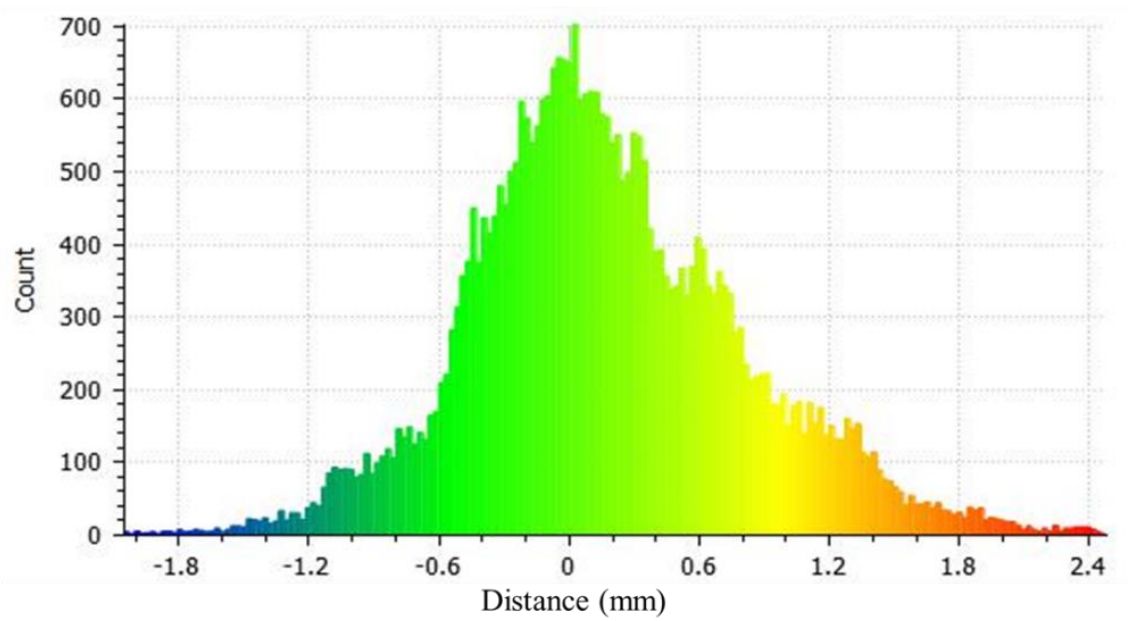

Fig. 6. Model showing the differences between the models as hypsometric colours. Right view (a), left (b), top (c) and back (d). 
The differences between the ellipsoidal semiaxes (Table 4) are $1 \mathrm{~mm}$ for two of the semiaxes, B and C (shortest); the difference for semiaxis A (longest) is $0.6 \mathrm{~mm}$. The precision of each semiaxis is between 0.02 and $0.03 \mathrm{~mm}$.

\begin{tabular}{llll}
\hline & Semiaxis $A(\mathrm{~mm})$ & Semiaxis $B(\mathrm{~mm})$ & Semiaxis $C(\mathrm{~mm})$ \\
\hline Smartphone & $68.9 \pm 0.02$ & $75.4 \pm 0.03$ & $82.3 \pm 0.03$ \\
SLR camera & $68.3 \pm 0.02$ & $74.4 \pm 0.03$ & $81.3 \pm 0.03$
\end{tabular}

Table 4. Fitted ellipsoid parameters for both models.

\section{DISCUSSION}

In medical applications, photography and photogrammetry are used for multiple purposes. However, its usage is not integrated as part of the consultation workflow and it is often related to high costs. One of these applications that we are envisioning is cranial deformation analysis, a condition that affects a great number of new-borns and that can vary from minor aesthetical problems (moderate positional plagiocephaly or brachiocephaly) to serious diseases such as craniosynostosis (Pathmanaban et al, 2016). The extensive usage of smartphone cameras for this cranial deformation analysis is especially interesting as the method is highly flexible and has an affordable (low) cost. The use of a slow-motion video recorded with a smartphone is especially suitable for this specific application as it works well for uncontrolled moving targets and reduces considerably the time required for the image acquisition process. In addition, no special lighting is required due to the high sensitivity of the smartphone cameras.

The creation of 3D models of moving infants using SLR cameras is challenging. It was possible in this particular case because the infant was calmer than normal (although moving). The problems encountered when using SLR cameras were in the same trend even while taking pictures with a high-end digital camera, such as the Canon 1Ds Mark III with a Canon lens EF $35 \mathrm{~mm}$ lens. New-borns and children below 6-8 months are unconsciously moving either in the consultancy or in the hospital.

In spite of their advantages, smartphone cameras -especially for slowmotion video- still have lower resolution than the $4 \mathrm{~K}$ video cameras and even the SLR cameras that are traditionally used for photogrammetric purposes. Smartphone cameras are also expected to have less stability on the interior orientation calibration, although this statement still needs to be confirmed from our side. 
Due to the demanding requirements in medical applications, it is vital to evaluate the accuracy and reliability of smartphone cameras for 3D modelling. In this study, we have compared two totally different cameras. In the first place, a professional, high-end SLR camera with a resolution of 4272 $x 2848$ pixels which requires the target to be still and needs a large amount of time to take the photographs. In the second place, a smartphone in slowmotion video mode recording, that despite its lower resolution $(1280 \times 738$ pixels), allows users to take enough imagery data in shorter time slots.

The camera self-calibration results show large errors, especially for the smartphone camera. The main limitation to obtain accurate calibration parameters is, undoubtedly, the geometry of the network. In this study, only a small area in the centre of the images was used. Moreover, not many accurate control points could be identified in the images. The identifiable points are concentrated on the measuring tape used as targets and the geometric distribution is far from ideal. For this reason, the calibration parameters need to be extrapolated providing very poor results. This situation is a limitation given by the image acquisition situation. After this experimentation, a new data acquisition framework was figured out: the implementation of an independent on-the-job calibration framework using images acquired by the doctor prior to the video recording. The accuracy improvement expected with a calibration framework will be studied in the future in case higher accuracy is requested for the image-based 3D modelling solution. The estimates of the additional calibration parameters were notably worse for the smartphone-based camera. This can be explained by the lack of stability of the video camera, and it is well-known from literature (Fraser, 1997).

The image measurement standard error is $0.016 \mathrm{~mm}$ for the SLR camera, and slightly higher for the smartphone camera, $0.02 \mathrm{~mm}$. However, the video allows the acquisition of a larger quantity of images in shorter periods of time resulting in a better estimated precision for the smartphone model. The precision achieved is $0.013 \mathrm{~mm}$ for the smartphone model and $0.019 \mathrm{~mm}$ for the SLR camera model. These results show that the differences in the estimated precision between the two methodologies are not relevant.

The mean Euclidean distance between the registered point clouds is $0.5 \mathrm{~mm}$. The mean of the signed distances is $0.17 \mathrm{~mm}$. With our large dataset (that included up to 34555 distances), this difference is significant and therefore it cannot be stated that models are the same. Nevertheless, the differences are small in the context of this application, as cranial deformation is usually measured using callipers that have a maximum accuracy of $1 \mathrm{~mm}$ in ideal conditions (rarely achieved in real clinical situations), without considering the landmarks identification error. Similar levels of accuracy are reported by other 
authors using high-cost special devices (Aldridge et al., 2005; Schaaf et al., 2010).

The differences in the semiaxis of the fitted ellipsoids have a maximum of 1 $\mathrm{mm}$, which is within tolerance with the accuracy of the parameters. These two latter evaluations coming from the Euclidean distances as well as from the semiaxes comparison allow us to confirm that the theoretical precision estimations presented in Table 3 are too optimistic.

Smartphone cameras in slow-motion mode are proven to be as suitable as SLR cameras for the creation of 3D models to evaluate cranial deformation in infants. In addition, smartphone slow-motion video reduces the time required to collect the images and produces even comparable results for analysing infants' head in movement.

\section{CONCLUSIONS}

The use of smartphone cameras, especially those with slow motion video mode, were proved to be a suitable technique for the creation of 3D models intended to evaluate cranial deformation in infants at close ranges. Although SLR cameras provide a slightly better standard deviation for image measurements, the slow-motion video images taken with a smartphone allow users the acquisition of a higher number of well-focused images at lower resolution, and at closer ranges (ca. $30 \mathrm{~cm}$ ). Besides, neither additional lighting is requested to take neat images with low ISO numbers nor additional accessories such as tripod are required. After this research, it can be confirmed that smartphones can provide similar precision to SLR cameras, but with higher flexibility and ability to move around during data acquisition, at low-cost and without carrying external lighting equipment. Moreover, the concept of slow motion video works better than still images to achieve focused images for uncontrolled moving targets. The use of single 3D scanners is discarded for this application, as they are incompatible with infant's movement.

\section{ACKNOWLEDGEMENTS}

The authors acknowledge the partial support from the Subprogram C (No. C10) to the development of coordinated actions between the Universitat Politècnica de València and the Hospital Universitari i Politécnic/Instituto de Investigación Sanitaria La Fe. 
Aldridge, K., Boyadjiev, S. A., Capone, G. T., DeLeon, V. B. and Richtsmeier, J. T. (2005) 'Precision and error of three-dimensional phenotypic measures acquired from 3dMD photogrammetric images', American Journal of Medical Genetics, 138 A(3), pp. 247-253. doi:

10.1002/ajmg.a.30959.

Bakula, K. and Flasiński, A. (2014) 'Capabilities of a smartphone for georeferenced 3D model creation: an evaluation'. In SGEM2014 Conference Proceedings, Book 2, Vol. 3, 85-92 pp. DOI 10.5593/SGEM2014/B23/S10.011Barbero-García, I., Lerma, J. L., Marqués-Mateu, Á. and Miranda, P. (2017) 'Low-Cost Smartphone-Based Photogrammetry for the Analysis of Cranial Deformation in Infants', World Neurosurgery, 102, pp. 545-554. doi: 10.1016/j.wneu.2017.03.015.

Bektas, S. (2015) 'Least square fitting of ellipsoid using orthogonal distances', Boletim de Ciências Geodésicas. Universidade Federal do Paraná, 21(2), pp. 329-339. doi: 10.1590/S1982-21702015000200019.

Bergerhoff, W. (1957) 'Roentgenogram of basilar impression evaluated by measurement.', Zentralblatt fur Neurochirurgie, 18(2-3), pp. 149-162.

Black, J. T. and Pappa, R. S. (2003) 'Videogrammetry Using Projected Circular Targets: Proof-of-Concept Test'. Available at: https://ntrs.nasa.gov/search.jsp?R=20030062249 (Accessed: 23 May 2017).

Byvaltsev, V., Belykh, O. and Belykh, E. (2012) 'New Aspects in the Epidemiology of Craniofacial Anomalies', World Neurosurgery, 77(5), pp. 599-600. doi: 10.1016/j.wneu.2012.03.003.

Chong, A. K. (2012) 'Exploiting HD Camcorders for Close-Up Human Movement Applications', The Photogrammetric Record. Blackwell Publishing Ltd, 27(138), pp. 227-237. doi: 10.1111/j.1477-9730.2012.00673.x.

Daponte, P., De Vito, L., Picariello, F. and Riccio, M. (2013) 'State of the art and future developments of measurement applications on smartphones', Measurement, 46(9), pp. 3291-3307. doi: 10.1016/j.measurement.2013.05.006.

Farnood Ahmadi, F. and Layegh, N. F. (2014) 'Integration of close range photogrammetry and expert system capabilities in order to design and implement optical image based measurement systems for intelligent diagnosing disease', Measurement, 51, pp. 9-17. doi:

10.1016/j.measurement.2014.01.019.

Fraser, C. S. (1992) 'Photogrammetric measurement to one part in a million', Photogrammetric Engineering and Remote Sensing, 58(3), pp. 305310. Available at: http://cat.inist.fr/?aModele=afficheN\&cpsidt=5056984.

Fraser, C. S. (1996) 'Network design', in Atkinson, K. B. (ed.) CloseRange photogrammetry and Machine Vision. Caithness, Scotland: Whittles, pp. 256-281.

Fraser, C. S. (1997) 'Digital camera self-calibration', ISPRS Journal of 
Photogrammetry and Remote Sensing, 52(4), pp. 149-159. doi: http://dx.doi.org/10.1016/S0924-2716(97)00005-1.

Grenness, M. J., Osborn, J. E. and Tyas, M. J. (2005) 'Stereophotogrammetric mapping of tooth replicas incorporating texture', Photogrammetric Record, 20(110), pp. 147-161. doi: 10.1111/j.14779730.2005.00311.x.

Hernandez, A. and Lemaire, E. (2016) 'A smartphone photogrammetry method for digitizing prosthetic socket interiors.', Prosthetics and orthotics international, pp. 1-5. doi: 10.1177/0309364616664150.

Kraus, K. (1997) Photogrammetry: Advanced methods and applications. $\mathrm{D}\{$ ü\}mmler (Photogrammetry / Karl Kraus). Available at: https://books.google.es/books?id=sih2QgAACAAJ.

Lin, S.-Y., Mills, J. P. and Gosling, P. D. (2008) 'Videogrammetric monitoring of as-built membrane roof structures', The Photogrammetric Record. Blackwell Publishing Ltd, 23(122), pp. 128-147. doi: 10.1111/j.1477-9730.2008.00477.x.

Pindrik, J., Molenda, J., Uribe-Cardenas, R., Dorafshar, A. H. and Ahn, E. S. (2016) 'Normative Ranges of Anthropometric Cranial Indices and Metopic Suture Closure during Infancy', Journal of Neurosurgery: Pediatrics, 18(December), pp. 667-673. doi: 10.3171/2016.5.PEDS14336.

Plank, L. H., Giavedoni, B., Lombardo, J. R., Geil, M. D. and Reisner, A. (2006) 'Comparison of infant head shape changes in deformational plagiocephaly following treatment with a cranial remolding orthosis using a noninvasive laser shape digitizer.', The Journal of craniofacial surgery, 17(6), pp. 1084-1091. doi: 10.1097/01.scs.0000244920.07383.85.

Rogers, G. F. (2011) 'Deformational Plagiocephaly, Brachycephaly, and Scaphocephaly. Part I', Journal of Craniofacial Surgery, 22(1), pp. 9-16. doi: 10.1097/SCS.0b013e3181f6c313.

Salazar-Gamarra, R., Seelaus, R., da Silva, J. V. L., da Silva, A. M. and Dib, L. L. (2016) 'Monoscopic photogrammetry to obtain 3D models by a mobile device: a method for making facial prostheses.', Journal of otolaryngology - head \& neck surgery = Le Journal d'oto-rhino-laryngologie et de chirurgie cervico-faciale. BioMed Central, 45(1), p. 33. doi:

10.1186/s40463-016-0145-3.

Schaaf, H., Malik, C. Y., Streckbein, P., Pons-Kuehnemann, J., Howaldt, H.-P. and Wilbrand, J.-F. (2010) 'Three-dimensional photographic analysis of outcome after helmet treatment of a nonsynostotic cranial deformity.', The Journal of craniofacial surgery. United States, 21(6), pp. 1677-1682. doi: 10.1097/SCS.0b013e3181f3c630.

Siegenthaler, M. H. (2015) 'Methods to Diagnose, Classify, and Monitor Infantile Deformational Plagiocephaly and Brachycephaly: A Narrative Review', Journal of Chiropractic Medicine. National University of Health 
Sciences, 14(3), pp. 191-204. doi: 10.1016/j.jcm.2015.05.003.

$\mathrm{Xu}, \mathrm{Z}$., Wu, T. H., Shen, Y. and Wue, L. (2016) 'Three dimentional reconstruction of large cultural heritage objects based on uav video and tls data', International Archives of the Photogrammetry, Remote Sensing and Spatial Information Sciences - ISPRS Archives, 41(July), pp. 985-988. doi: 10.5194/isprsarchives-XLI-B5-985-2016. 